\title{
USE OF GFP FOR IN VIVO IMAGING: CONCEPTS AND MISCONCEPTIONS
}

\author{
Robert M. Hoffman \\ AntiCancer, Inc., 7917 Ostrow Street, San Diego, CA 92111-3604 \\ Dept. of Surgery, University of California, 200 West Arbor Drive, San Diego, CA 92103-8220
}

\begin{abstract}
Although GFP and fluorescent proteins are used extensively for in vivo imaging, there are many misconceptions about GFP imaging especially compared to luciferase. GFP is not toxic, indeed, transgenic animals with GFP expressed in every cell (1) live as long as non-transgenic animals. Cancer cells with GFP are as aggressive and malignant as the cells without GFP (2-4). Cell lines can be made very bright with fluorescent proteins with no toxicity. The in vivo signal from fluorescent proteins is at least 1,000 times greater than luciferase (5). GFP is so bright that a single molecule of GFP can be seen in a bacterium (6). GFP can be observed through the skin on deep organs (7). Skin autofluorescence presents no problem for in vivo GFP imaging with proper filters (8). Fur can be rapidly clipped removing this autofluorescence (9). GFP is readily quantified by the image area which correlates to tumor volume (10). There are now numerous clones of GFP, RFP, YFP and proteins that change color (11) that can be used in vivo.
\end{abstract}

Keywords: green fluorescent protein, mice, imaging, non-invasive, autofluorescence

\section{INTRODUCTION}

Naturally fluorescent proteins have revolutionized biology by enabling what was formerly invisible to be seen clearly in the living state. These proteins have allowed us to visualize, in real time, important aspects of cancer in living animals, including tumour cell mobility, invasion, metastasis and angiogenesis. These multicoloured proteins have allowed the colour-coding of cancer cells growing in vivo and enabled the distinction of host from tumour with single-cell resolution. Visualization of many aspects of cancer progression in vivo has been accomplished noninvasively as well as invasively with fluorescent proteins (2).

To image and follow the natural course or impediment of tumor progression and metastasis, high specificity and sensitivity, a strong signal, and high resolution are necessary. The GFP gene, cloned from the bioluminescent jellyfish Aequorea victoria (12), was chosen to satisfy these conditions because it has great potential for use as a cellular marker (13). GFP cDNA encodes a 283-amino acid monomeric polypeptide with $M \mathrm{r} 27,000(14,15)$ that requires no other $A$. victoria proteins, substrates, or cofactors to fluoresce (16). Gain-of-function bright mutants expressing the GFP gene have been generated by various techniques $(17,18)$ and have been humanized for high expression and signal $(19)$. Red fluorescent proteins (RFP) from the Discosoma coral and other organisms have similar features as well as the advantage of longer-wavelength emission (20).

\section{MISCONCEPTIONS}

Despite the numerous published reports on the efficiency of imaging with fluorescent proteins, there are comments in the literature denying these facts. Some examples are quoted below:

“. . . fluorescence imaging still suffers from pitfalls such as the inability to quantify photon output, high autofluorescence in the blue-green window resulting in low signal-to-noise ratios, fluorophore photo-bleaching, and high levels of photon attenuation and scattering in living tissues" (21).

Small Animal Whole-Body Optical Imaging Based on Genetically Engineered Probes edited by Alexander P. Savitsky, Robert E Campbell, Robert M. Hoffman

Proc. of SPIE Vol. 6868, 68680E, (2008) - 1605-7422/08/\$18 - doi: 10.1117/12.774181

Proc. of SPIE Vol. 6868 68680E-1 
"Photon attenuation, however, is strongly nonlinear as a function of depth and of the optical heterogeneity of tissue, which obscures signal quantification. Planar imaging is further complicated by the inability to resolve depth and by tissue scattering, which limits spatial resolution. For these reasons, although planar methods are useful, they do not harness the true potential of the optical imaging technologies" (22).

"Yang et al. (Proc. Natl. Acad. Sci. USA 97, 1206-1211, 2000), for example, demonstrated that the use of a highly sensitive color CCD camera can detect green fluorescent proteins expressed by tumors implanted superficially in living animals" (22).

"However, it (planar GFP imaging in vivo) also has important limitations, such as the single projection viewing, the restricted penetration depth of a few millimeters and the nonlinear relationship between the signal strength and the depth and the tissue optical properties. These features limit the applicability of the method primarily to superficial observations and may lead to erroneous interpretation of the data collected if the nonlinear effects are not explicitly corrected or accounted for" (22).

"Although GFP imaging of surface tumors is feasible and experimentally useful, deep-seated tumors and organ structures have to be accessed surgically for observation" (23).

“... fluorescent techniques have tremendous utility in the study (of) cells in culture, excised tissues, small transparent organisms, or biological processes that occur at superficial tissue sites in mammals" (24).

"A drawback of GFP is its low emission wavelength $(\sim 510 \mathrm{~nm})$, which overlaps with the autofluorescence of many tissues" (23).

"In fluorescent imaging, the excitation light also will excite naturally occurring fluors in the tissue (autofluorescence) in addition to the fluorescent tag. This is the source of noise in FLI (fluorescence imaging), and it can be significant, especially at shorter wavelengths of light (300 to $600 \mathrm{~nm}$ )" (24).

"The wavelengths of light that are needed to excite many of the fluors that have been developed for use in microscopy and cytometry are in the blue and green region of the visible spectrum. Light of these wavelengths has limited penetration through mammalian tissues because of absorption, which is, in large part, caused by hemoglobin" (24).

"Noise in fluorescent imaging arises from the autofluorescence of tissue (this is not the case with reporter genes that encode bioluminescent proteins, because there is little, or essentially no, background autoluminescence), and once a reporter gene is expressed, the signal depends on the absorbing and scattering properties of the tissue, which are greater with shorter wavelengths of light" (24).

"At present, the primary disadvantage of fluorescence, compared to bioluminescence, is the presence of background signal from auto-fluorescence of innate biological molecules" (25).

\section{CONCEPTS}

However, in contrast to the above published statements, we describe below specific examples demonstrating that in vivo imaging with fluorescent proteins is far superior to in vivo imaging with luciferase.

A triple fusion reporter vector containing a luciferase gene, red fluorescence protein gene and a thymidine kinase gene was found to highly express all three genes. Mice implanted with 293T cells expressing the vector were imaged with a highly sensitive cooled charge-coupled device camera compatible with both luciferase and fluorescence imaging. When the quantitative data in this report are analyzed, it can be seen that the intensity of photon emission from RFP expression in the tumor cells in mice is at least 1,000 times greater than that of luciferase (5).

Indeed, fluorescent proteins are so bright that a single molecule of GFP can be detected as bacteria. A fusion protein of a fast-maturing yellow fluorescent protein (YFP) and a membrane-targeting peptide was expressed under a repressed condition. The membrane-localized YFP can be detected with single-molecule sensitivity. It was found that the protein 
molecules are produced in bursts, with each burst originating from a stochastically transcribed single messenger RNA molecule, and that protein copy numbers in the bursts follow a geometric distribution (6).

In 2000, we initially demonstrated that we could noninvasively image, in real time, GFP-expressing tumors growing and metastasizing in live mice. The whole-body optical imaging system is external and noninvasive. Noninvasive GFP imaging enables unprecedented continuous visual monitoring of malignant growth and spread within intact animals. We have established human and rodent tumors that stably express very high levels of the Aequorea victoria GFP and transplanted these to appropriate animals. B16F0-GFP mouse melanoma cells were injected into the tail vein or portal vein of 6-week-old C57BLy6 and nude mice. Whole-body optical images showed metastatic lesions in the brain, liver, and bone of B16F0-GFP that were used for real time, quantitative measurement of tumor growth in each of these organs. The AC3488-GFP human colon cancer was surgically implanted orthotopically into nude mice. Whole-body optical images showed, in real time, growth of the primary colon tumor and its metastatic lesions in the liver and skeleton. Imaging was with either a trans-illuminated epifluorescence microscope or a fluorescence light box and thermoelectrically cooled color charge-coupled device camera (7).

A blue LED flashlight (LDP LLC, Woodcliff Lake, NJ, USA; www.maxmax.com/OpticalProducts.htm) with an excitation filter (midpoint wavelength peak of $470 \mathrm{~nm}$ ) and an emission D470/40 filter (Chroma Technology, Brattleboro, VT, USA) for viewing was used for whole-body imaging of mice with GFP and RFP-expressing tumors growing in or on internal organs. The images were readily seen by the naked eye with no anesthesia, substrate, or restraint of the animal needed. Even more striking was that the intensity of the whole-body image is $70 \%$ of the open image. Although some information is lost with whole-body imaging due to light scattering, a remarkable amount of information is obtained, even with such simple instrumentation. No skin autofluorescence was found due to the use of the proper excitation filter (8).

There have also been reports that GFP is toxic $(26,27)$. In this light, it should be noted that transgenic mice have been produced which express GFP in essentially every cell. These mice live as long as wild-type mice (1), demonstrating no toxicity from GFP. The transgenic mouse lines were produced with a GFP/cDNA under the control of a chicken betaactin promoter and cytomegalovirus enhancer. All of the tissues from these transgenic lines, with the exception of erythrocytes and hair, were green under excitation light (1).

In addition, the in vivo metastatic behavior of cancer cells with and without GFP have been compared: MDA-MB-435 human breast cancer cells engineered with GFP were injected into the cardiac left ventricle of athymic mice. Femurs were analyzed by fluorescence microscopy, immunohistochemistry, real-time PCR, flow cytometry, and histomorphometry at times ranging from 1 hour to 6 weeks. Single cells were found in distal metaphyses at 1 hour postinjection and remained as single cells up to 72 hours. At 1 week, numerous foci (2-10 cells) were observed, mostly adjacent to osteoblast-like cells. By 2 weeks, fewer but larger foci ( $>$ or $=50$ cells) were seen. Most bones had a single large mass at 4 weeks (originating from a colony or coalescing foci) which extended into the diaphysis by 4 to 6 weeks (3). GFP-tagging permitted detection of single MDA-MB-435 cells and microscopic metastases in bone at early time points following arrival and at stages of proliferation prior to coalescence of individual metastases (4).

There are also reports that GFP is immunogenic (28-30). However, studies of syngenic mice with GFP tumors show the tumors can be highly malignant, suggesting that any antigenicity of GFP did not affect malignancy (9).

Despite the published statements listed above that imaging with fluorescent proteins is non-quantitative, it is not the case. A highly fluorescent RFP-expressing pancreatic cancer model was orthotopically established in nude mice with the MIA-PaCa-2 human pancreatic cancer cell line. Rapid tumor growth and widespread metastases developed in the mice within two weeks, leading to a median survival of 21 days. The noninvasive imaging of the RFP-expressing tumor and metastases was compared to tumor volume measurements with high correlation. Thus, noninvasive, sequential imaging with fluorescent permits quantification of tumor growth and dissemination (10). 


\section{THE FUTURE}

\subsection{New fluorescent proteins}

New fluorescent proteins have been isolated, some that are far red shifted, which will make noninvasive imaging even more powerful.

Labas et al. have isolated 11 new fluorescent protein genes, among which there are three red-emitters. Phylogenetic analysis has shown that GFP-like proteins from representatives of subclass Zoantharia fall into at least four distinct clades, each clade containing proteins of more than one emission color. This suggests multiple recent events of color conversion. Combining this result with previous mutagenesis and structural data, Labas et al. propose that (i) different chromophore structures are alternative products synthesized within a similar autocatalytic environment, and (ii) the phylogenetic pattern and color diversity in reef Anthozoa is a result of a balance between selection for GFP-like proteins of particular colors and mutation pressure driving the color conversions. These data suggest many types of fluorescent proteins will be discovered in the near future (11).

Fluorescent proteins with distinguishable colors from yellow-orange to red-orange have now been developed by mutagenesis (31).

A far-red fluorescent protein, named Katushka, which is seven- to ten-fold brighter compared to proteins with similar emission has been developed. Katushka appears to be highly resistant to photo-bleaching. Katushka was shown to be superior for whole-body imaging by direct comparison with other red and far-red fluorescent proteins (32).

\subsection{New imaging systems}

Recently the Olympus OV100 whole-mouse imaging system, with a sensitive CCD camera and four objective lenses, parcentered and parfocal, enabling imaging from macrocellular to subcellular, has been developed. With this instrument, we have observed the nuclear and cytoplasmic behavior of cancer cells in real time in blood vessels as they moved by various means or adhered to the vessel surface in the abdominal skin flap. During extravasation, real-time dual-color imaging showed that cytoplasmic processes of the cancer cells exited the vessels first, with nuclei following along the cytoplasmic projections. Both cytoplasm and nuclei underwent deformation during extravasation. Different cancer cell lines seemed to strongly vary in their ability to extravasate. With the dual-color cancer cells and the highly sensitive whole-mouse imaging system described here, the subcellular dynamics of cancer metastasis can now be observed in live mice in real time (33).

\subsection{Fluorescent proteins can enable non-invasive imaging at the subcellular level.}

To noninvasively image cancer cell/stromal cell interaction in the tumor microenvironment and drug response at the cellular level in live animals in real time, we developed a new imageable three-color animal model. The model consists of GFP-expressing mice transplanted with dual-color cancer cells labeled with GFP in the nucleus and red fluorescent protein in the cytoplasm. The Olympus IV100 Laser Scanning Microscope, with ultra-narrow microscope objectives ("stick objectives"), is used for three-color whole-body imaging of the two-color cancer cells interacting with the GFPexpressing stromal cells. In this model, drug response of both cancer and stromal cells in the intact live animal is also imaged in real time. Various in vivo phenomena of tumor-host interaction and cellular dynamics were imaged, including mitotic and apoptotic tumor cells, stromal cells interacting with the tumor cells, tumor vasculature, and tumor blood flow. This new model system enables the first cellular and subcellular images of unperturbed tumors in the live intact animal. New visible real-time targets for novel anticancer agents are provided in this model, including the colorcoded interacting cancer and stromal cells, tumor vasculature, and blood flow. This imageable model should lead to many new insights of in vivo cancer cell biology and to novel drug discovery. These data therefore demonstrate that single cells expressing fluorescent proteins can be imaged noninvasively (34).

Fluorescent protein imaging will enable further understanding of the critical steps of metastasis and provide visible targets for antimetastasis drug development. In conclusion, new red-shifted proteins and superior imaging equipment will enable fluorescent imaging technology to develop the new field of in vivo cell biology. 


\section{REFERENCES}

1. M. Okabe, M. Ikawa, K. Kominami, T. Nakanishi, Y. Nishimune, "Green mice' as a source of ubiquitous green cells", FEBS Lett. 407, pp. 313-319, 1997.

2. $\quad$ R.M. Hoffman, "The multiple uses of fluorescent proteins to visualize cancer in vivo”, Nat. Rev. Cancer 5, pp. 796-806, 2005.

3. P.A. Phadke, R.R. Mercer, J.F. Harms, Y. Jia, A.R. Frost, J.L. Jewell, K.M. Bussard, S. Nelson, C. Moore, J.C. Kappes, C.V. Gay, A.M. Mastro, D.R. Welch, "Kinetics of metastatic breast cancer cell trafficking in bone", Clin. Cancer Res. 12, pp. 1431-1440, 2006.

4. J.F. Harms, D.R. Welch, "MDA-MB-435 human breast carcinoma metastasis to bone," Clin. Exp. Metastasis 20, pp. 327-334, 2003.

5. P. Ray, A De, J.J. Min, R.Y. Tsien, S.S. Gambhir, "Imaging tri-fusion multimodality reporter gene expression in living subjects," Cancer Res. 64, 1323-1330, 2004.

6. J. Yu, J. Xiao, X. Ren, X., K. Lao, X.S. Xie, "Probing gene expression in live cells, one protein molecule at a time," Science 311, pp. 1600-1603, 2006.

7. M. Yang, E. Baranov, P. Jiang, F-X. Sun, X-M. Li, L. Li, S. Hasegawa, M. Bouvet, M. Al-Tuwaijri, T. Chishima, H. Shimada, A.R. Moossa, S. Penman, R.M. Hoffman, "Whole-body optical imaging of green fluorescent protein-expressing tumors and metastases", Proc. Natl. Acad. Sci. USA 97, pp. 1206-1211, 2000.

8. M. Yang, G. Luiken, E. Baranov, R.M. Hoffman, "Facile whole-body imaging of internal fluorescent tumors in mice with an LED flashlight", BioTechniques 39, pp. 170-172, 2005.

9. C.A. Schmitt, J.S. Fridman, M. Yang, E. Baranov, R.M. Hoffman, S.W. Lowe, "Dissecting p53 tumor suppressor functions in vivo", Cancer Cell 1, pp. 289-298, 2002.

10. M. Katz, S. Takimoto, S., D. Spivac, A.R. Moossa, R.M. Hoffman, M. Bouvet, "A novel red fluorescent protein orthotopic pancreatic cancer model for the preclinical evaluation of chemotherapeutics", J. Surg. Res. 113 , pp. 151-160, 2003.

11. Y.A. Labas, N.G. Gurskaya, Y.G. Yanushevich, A.F. Fradkov, K.A. Lukyanov, S.A. Lukyanov, M.V. Matz, "Diversity and evolution of the green fluorescent protein family", Proc. Natl. Acad. Sci. USA 99, pp. 42564261, 2002.

12. D.C. Prasher, V.K. Eckenrode, W.W. Ward, F.G. Prendergast, M.J. Cormier, "Primary structure of the Aequorea victoria green-fluorescent protein," Gene 111, pp. 229-233, 1992..

13. M. Chalfie, Y. Tu, G. Euskirchen, W.W. Ward, D.C. Prasher," Green fluorescent protein as a marker for gene expression", Science 263, pp. 802-805, 1994.

14. C.W. Cody, D.C. Prasher, W.M. Westler, F.G. Prendergast, W.W. Ward, "Chemical structure of the hexapeptide chromophore of the Aequorea green fluorescent protein”, Biochemistry 32, pp. 1212-1218, 1993.

15. F. Yang, L.G. Moss, G.N. Phillips Jr., "The molecular structure of green fluorescent protein”, Nat Biotechnol. 14, pp. 1246-1251, 1996.

16. J. Morin, J. Hasting, “Energy transfer in a bioluminescent system,” J. Cell. Physiol. 77, pp. 313-318, 1971. 
17. B. Cormack, R. Valdivia, S. Falkow, "FACS-optimized mutants of the green fluorescent protein (GFP)," Gene 173, pp. 33-38, 1996.

18. A. Crameri, E.A. Whitehorn, E. Tate, W.P. Stemmer, "Improved green fluorescent protein by molecular evolution using DNA shuffling," Nat. Biotechnol. 14, pp. 315-319, 1996.

19. R. Heim, A.B. Cubitt, R.Y. Tsien, "Improved green fluorescence,” Nature 373, pp. 663-664, 1995.

20. A.F. Fradkov, Y. Chen, L. Ding, E.V. Barsova, M.V. Matz, S.A. Lukyanov, "Novel fluorescent protein from Discosoma coral and its mutants possesses a unique far-red fluorescence," FEBS Lett. 479, pp. 127-30, 2000.

21. S. Gross, D. Piwnica-Worms, "Spying on cancer: Molecular imaging in vivo with genetically encoded reporters," Cancer Cell 7, pp. 5-15, 2005.

22. V. Ntziachristos, J. Ripoll, L.V. Wang, R. Weissleder, "Looking and listening to light: the evolution of wholebody photonic imaging," Nat. Biotechnol. 23(3), pp. 313-320, 2005.

23. R. Weissleder, V. Ntziachristos, "Shedding light onto live molecular targets," Nat. Med., 9(1), pp. 123-128, 2003.

24. C. Contag, "Molecular imaging using visible light to reveal biological changes in the brain," Neuroimaging Clinics of North America 16(4), pp. 633-654, 2006.

25. R. Paulmurugan, P. Ray, A. De, C.T. Chan, S.S. Gambhir, "Imaging protein-protein interactions in living subjects," Trends in Analytical Chem., 24, pp. 446-458, 2005.

26. B. Badrian, M.A. Bogoyevitch," "Changes in the transcriptional profile of cadiac myocytes following green fluorescent protein expression," DNA and Cell Biology 26, pp. 727-735, 2007.

27. H.S. Liu, M.S. Jan, C.K. Chou, P.H. Chen, N.J. Ke, "Is green fluorescent protein toxic to the living cells?" Biochem. Biophys. Res. Commun. 260, pp. 712-717, 1999.

28. R. Stripecke, M. Carmen Villacres, D. Skelton, N. Satake, S. Halene, D. Kohn, "Immune response to green fluorescent protein: implications for gene therapy", Gene Ther. 6, 1305-1312, 1999.

29. K. Yamamoto, K. Yuasa, Y. Miyagoe, Y, Hosaka, K. Tsukita, H. Yamamoto, Y.I. Nabeshima, S. Takeda, "Immune response to adenovirus-delivered antigens upregulates utrophin and results in mitigation of muscle pathology in mdx mice," Hum. Gene Ther. 11, pp. 669-680, 2000.

30. K.E. Beagles, L. Peterson, X. Zhang, J. Morris, H.P. Kiem, "Cyclosporine inhibits the development of green fluorescent protein (GFP)-specific immune responses after transplantation of GFP-expressing hematopoietic repopulating cells in dogs," Hum. Gene Ther. 16, pp. 725-733, 2005.

31. N.C. Shaner, P.A. Campbell, B.N. Giepmans, A.E. Palmer, R.Y. Tsien, "Improved monomeric red, orange and yellow fluorescent proteins derived from Discosoma sp. Red fluorescent protein", Nat. Biotechnol. 22, pp. $1567-1572,2004$.

32. D. Shcherbo, E.M. Merzlyak, T.V. Chepurnykh, A.F. Fradkov, G.V. Ermakova, E.A. Solovieva, K.A. Lukyanov, E.A. Bogdanova, A.G. Zaraisky, S. Lukyanov, D.M. Chudakov, "Bright far-red fluorescent protein for whole-body imaging," Nat. Methods. 4, pp. 741-746, 2007.

33. K. Yamauchi, M. Yang, P. Jiang, M. Xu, N. Yamamoto, H. Tsuchiya, K. Tomita, A.R. Moossa, M. Bouvet, R.M. Hoffman, "Development of real-time subcellular dynamic multicolor imaging of cancer cell trafficking in live mice with a variable-magnification whole-mouse imaging system," Cancer Res. 66, pp. 4208-4214, 2006. 
34. M. Yang, P. Jiang, R,M. Hoffman, "Whole-body subcellular multicolor imaging of tumor-host interaction and drug response in real time", Cancer Res. 67, pp. 5195-5199, 2007. 